\title{
Social Assistance in Developing Countries
}

by Armando Barrientos, Professor and Research Director of the Brooks World Poverty Institute at the University of Manchester

In a comparatively short period of time, programmes providing direct transfers in cash and in kind to households in poverty have expanded to reach between 740 million and 1 billion people in the South. The book Social Assistance in Developing Countries provides a comprehensive account of the global growth of social assistance transfer programmes in developing countries.

Social assistance describes programmes and policies addressing poverty and vulnerability. In the 'golden age' of European welfare states, social assistance was seen as a residual institution and expected to wither away. In the last two decades, developing countries have introduced or expanded innovative forms of social assistance contributing to the reduction of global poverty.

\section{The book is divided into two parts: foundations and practice.}

Part I develops a framework linking the ethics of assistance, poverty analysis, the economics of social assistance and institutional dynamics. It argues that assisting people in poverty can be grounded on a political notion of justice which gives priority to assisting disadvantaged groups with the aim of securing their full participation in society.

Together with a primer on poverty analysis, this informs a discussion on optimal transfer programmes. The economics of optimal transfer programmes suggests ways in which programme design features could improve the effectiveness of antipoverty transfers.

Part II focuses on existing practice in developing countries. Research aiming to take a comparative view of social assistance will face the challenge of organising the considerable diversity in programme design and objectives; therefore, classification into three categories is proposed: pure income transfers; transfers combined with asset accumulation; and integrated antipoverty programmes.

The classification is based on the understandings of poverty underpinning these programmes. Pure income transfers focus on income or consumption poverty. Transfers combined with asset accumulation are based on multidimensional perspectives on poverty. Integrated antipoverty programmes stress the role of social exclusion. This classification helps to analyse three key features of social assistance in the South: attention to incentives; packaging of interventions; and a focus on enhancing productive capacity.

A chapter is devoted to the three I's: incidence, implementation and impact. It notes the relative advantage of a process approach to beneficiary selection over 'targeting' approaches. It argues that greater attention needs to be paid to implementation issues than has been the case in the literature. Summarising the growing evidence on the impact of social assistance, it confirms the basis for a growing consensus on the effectiveness of well-designed and well-implemented programmes, alongside significant evidence gaps.

Researchers have paid scant attention to the politics of social assistance. A discussion of politics is linked to a discussion of financing. The dominant 'resource mobilisation' perspective favoured by donors is contrasted with a more rounded approach to financing which incorporates incentives and legitimacy into the financing mix. The financing options open to developing countries involve resource revenues and consumption taxes, each with distinct challenges.

Where do we go from here? The book argues that the best way to understand the growth of social assistance in developing countries is in terms of institution building.

Democratisation has placed a premium on the inclusion of low-income and vulnerable groups. Growth has expanded the fiscal space available to policymakers. The remarkable expansion of transfer programmes in the South signals a route to establishing strong and stable institutions in the South capable of reducing poverty to zero and keeping it there.

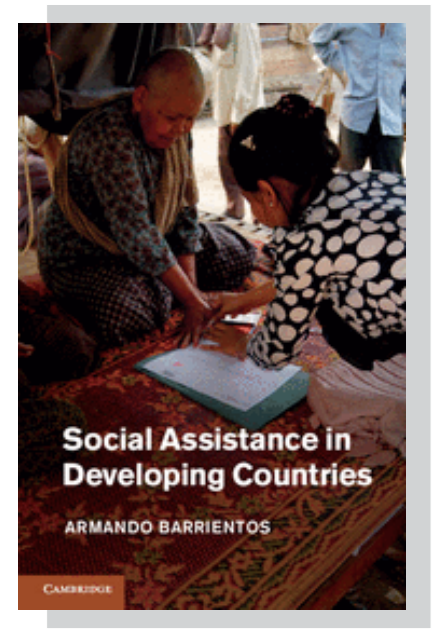

